\title{
Freiheitsgrad agiler Methoden versus QM-Anforderungen - eine Empfehlung
}

Spätestens mit den Fragestellungen rund um ,agile Unternehmen“ ist Agilität auch im Qualitätsmanagement als Thema angekommen. Freiheitsgrade, die agile Teams einfordern, kollidieren möglicherweise mit QM-Vorgaben, aber diese Konflikte lassen sich nach unserer Erfahrung meistens befriedigend auflösen. Wie dies jetzt und künftig gelingen kann, haben wir in diesem Beitrag dargelegt. Letztlich verfolgen beide Parteien die gleichen Ziele: reife Prozesse und hohe Produktqualität. Nur die Wege dorthin unterscheiden sich, denn sie kommen aus unterschiedlichen Richtungen - agile Teams „,bottom up“ und QM-Stellen „top down“. Unverzichtbar ist auf beiden Seiten die Bereitschaft, die Sichtweisen und Methoden der jeweils anderen Fraktion undogmatisch zu betrachten. Letztlich hilft auch hier einer der vier agilen Werte des zu Beginn zitierten Manifests: „,collaboration“ ist demnach ein höherer Wert als die sture „,contract negotiation“. 